\title{
Cryo-transmission electron tomography of native casein micelles from bovine milk
}

\author{
R. Trejo, ${ }^{*}$ T. Dokland, $\dagger$ J. Jurat-Fuentes, $\ddagger$ and F. Harte ${ }^{\star 1}$ \\ *Department of Food Science and Technology, The University of Tennessee, Knoxville 37996-4539 \\ †Department of Microbiology, University of Alabama at Birmingham, Birmingham 35294-2170 \\ ‡Department of Entomology and Plant Pathology, The University of Tennessee, Knoxville 37996-4539
}

\begin{abstract}
Caseins are the principal protein components in milk and an important ingredient in the food industry. In liquid milk, caseins are found as micelles of casein proteins and colloidal calcium nanoclusters. Casein micelles were isolated from raw skim milk by size exclusion chromatography and suspended in milk protein-free serum produced by ultrafiltration (molecular weight cut-off of $3 \mathrm{kDa}$ ) of raw skim milk. The micelles were imaged by cryo-electron microscopy and subjected to tomographic reconstruction methods to visualize the 3 -dimensional and internal organization of native casein micelles. This provided new insights into the internal architecture of the casein micelle that had not been apparent from prior cryo-transmission electron microscopy studies. This analysis demonstrated the presence of water-filled cavities ( $\sim 20$ to $30 \mathrm{~nm}$ in diameter), channels (diameter greater than $\sim 5 \mathrm{~nm}$ ), and several hundred high-density nanoclusters (6 to $12 \mathrm{~nm}$ in diameter) within the interior of the micelles. No spherical protein submicellar structures were observed.
\end{abstract}

Key words: casein micelle, micelle model, cryo-transmission electron tomography

\section{INTRODUCTION}

Caseins are the primary protein components in milk. These proteins form large colloidal particles, of 50 to $600 \mathrm{~nm}$ in diameter, known as casein micelles. Many of the technologically important properties of milk (e.g., white color, stability to heat or ethanol, and coagulation by rennet) are due to the properties of the casein micelles. Therefore, economic and technological incentives exist to characterize their properties and determine their structure (Fox and Brodkorb, 2008). The main biological function of the casein micelle is to deliver calcium from mother to offspring, as milk is

Received March 16, 2011.

Accepted August 16, 2011

${ }^{1}$ Corresponding author: fede@utk.edu supersaturated with calcium found in the casein micelle as colloidal calcium phosphate (CCP).

Several models were proposed to describe the nanostructure of the casein micelle, and recent detailed reviews can be found elsewhere (Qi, 2007; Farrell et al., 2006; Horne, 2009; Dalgleish, 2011), including a discussion on the use of the term "casein micelle" (Fox and Brodkorb, 2008). The casein micelle has been described as containing submicelles linked together by CCP (Walstra, 1999) or formed from CCP nanoclusters bound to phosphoserine-rich $\beta$ - and $\alpha$-casein proteins (De Kruif and Holt, 2003). The Horne model (Horne, 1998) considered the surface chemistry of the individual caseins and concluded that nonspecific protein-protein interactions also play a major role determining the casein micelle structure (Farrell et al., 2006). In most cases, the nanostructure of the casein micelle has been derived from x-ray or neutron scattering studies (Hansen et al., 1996; Holt et al., 2003) or by transmission or scanning electron microscopy observations (Marchin et al., 2007; McMahon and Oommen, 2008; Qi, 2009). A major hurdle in obtaining a definitive image of the native casein micelle through traditional electron microscopy techniques is the high water content of the micelles (an average of $3.7 \mathrm{~g}$ of water/g of casein protein; Wong et al., 1996). Conventional electron microscopy requires preparation methods that involve sample dehydration, fixation, and staining, all of which potentially introduce artifacts that are difficult to discriminate or quantify. In cryogenic-transmission electron microscopy (cryo-TEM), a thin layer of sample suspension is plunge-frozen in a suitable cryogenic fluid, resulting in the sample being embedded in a thin layer of vitreous ice, which retains the amorphous nature of the aqueous sample environment. Then, the sample is observed under cryogenic conditions, ensuring that the specimen is studied in a close-to-native state of preservation. Contrast in these specimens is generated not by the introduction of heavy atom stains, but through phasecontrast, which is produced from the coherent interference of the scattered and transmitted electron beams. Cryo-transmission electron tomography uses a series 
of cryo-TEM images taken in angular increments to assemble a 3-dimensional reconstruction of the object, including its internal features (Nudelman et al., 2011). The purpose of this study was to use cryo-TEM to visualize the internal architecture of native casein micelles in 3 dimensions.

\section{MATERIALS AND METHODS}

\section{Milk Source and Sample Preparation}

Raw milk samples were collected at a local dairy farm. Fat was removed by centrifugation at $4^{\circ} \mathrm{C}(6,414$ $\times g$ for $20 \mathrm{~min}$ ) and stored at $4^{\circ} \mathrm{C}$. Protein-free serum (PFS) was prepared by tangential flow ultra-filtration (TFUF) of pasteurized skim milk using a $3-\mathrm{kDa}$ mo- lecular weight cut-off cellulose filter (PLBC Prep scale TFF Cartridge, Millipore, Billerica, MA). The PFS was preserved with $0.07 \% \mathrm{NaN}_{3}$ and stored at $4^{\circ} \mathrm{C}$.

\section{Size Exclusion Chromatography}

A size exclusion chromatography column (Superdex 200 prep grade, GE Life Sciences, GE Health Care, Piscataway, NJ) connected to a fast performance liquid chromatography unit (AKTA, GE Life Sciences) was used for the isolation of casein micelles. Raw skim milk samples were loaded into the column, and ice-cold PFS (prepared as previously mentioned) was used for elution to preserve the native conditions. Separation was achieved using a flow rate of $1.0 \mathrm{~mL} / \mathrm{min}$ for 2 column volumes (240 $\mathrm{mL}$ total), collecting 2 -mL fractions. The

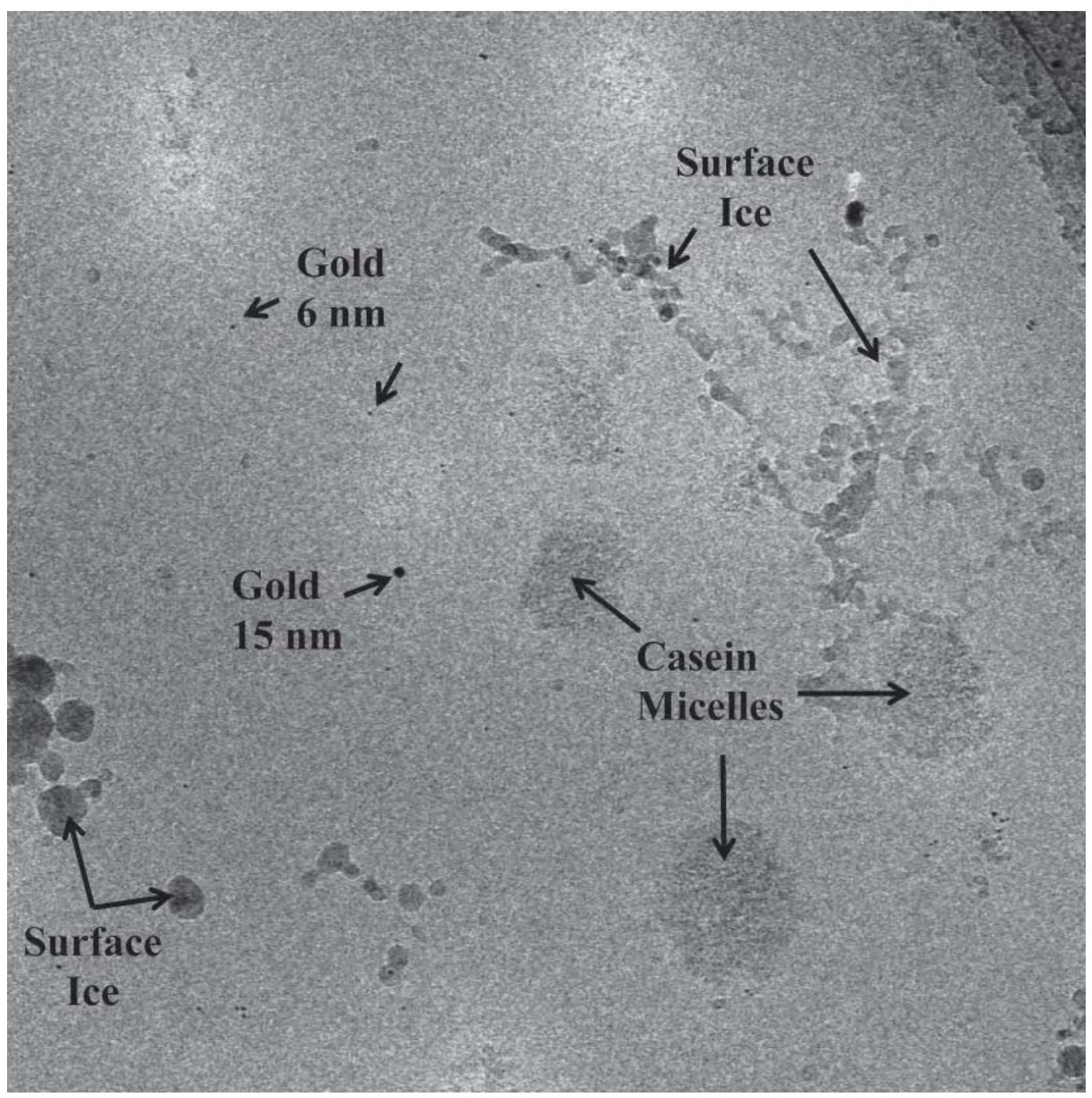

Figure 1. Cryo-transmission electron tomography image of native casein micelles. Three casein micelles, 6- and 15-nm gold particles, and ice contaminants are indicated. 


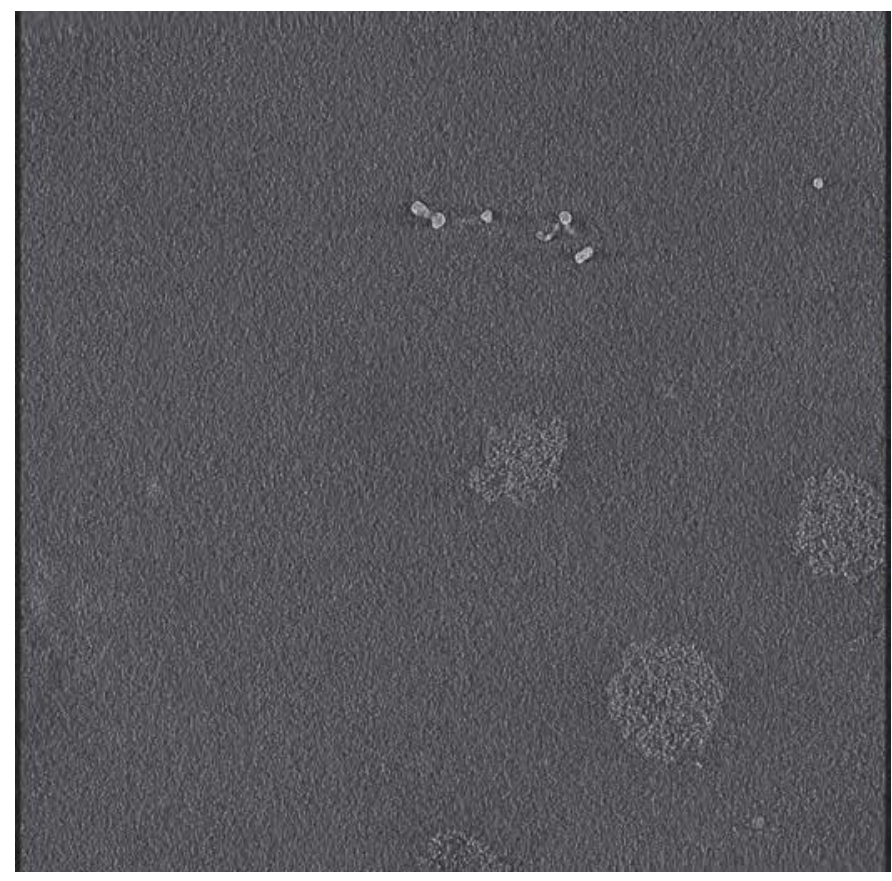

Figure 2. A 4.74-nm-thick slice through the center of one cryotransmission electron tomogram, showing 3 casein micelles. The contrast of the figure is inverted relative to the micrograph shown in Figure 1.

protein elution chromatograph was determined using absorbance set at $280 \mathrm{~nm}$. Collected fractions containing the casein micelles (confirmed by SDS-PAGE) were stored at $4^{\circ} \mathrm{C}$.

\section{Cryo-TEM}

Immediately before imaging by cryo-TEM, a sample was prepared containing $4 \mu \mathrm{L}$ of casein micelle isolates (from fast performance liquid chromatography), $18 \mu \mathrm{L}$ of PFS (permeate from 3.5-kDa TFUF of skim milk), $36 \mu \mathrm{L}$ of distilled water, $2 \mu \mathrm{L}$ of 6 -nm BSA-gold tracer (Electron Microscopy Sciences, Hatfield, PA), and $2 \mu \mathrm{L}$ of 15-nm Protein A-gold (Electron Microscopy Sciences). The gold particles were added as fiducial markers for the tomographic reconstruction. Three microliters of this suspension was pipetted onto 200-mesh holey grids (R2/1 Quantifoil Micro Tools, Jena, Germany), blotted briefly, frozen by plunging into liquid ethane at $-182^{\circ} \mathrm{C}$, transferred to a Gatan 626 cryo-sample holder (Gatan Inc., Pleasanton, CA), and observed in an FEI Tecnai F20 electron microscope (FEI, Hillsboro, OR) operated at $200 \mathrm{kV}$ (Dokland and Ng, 2006). Tomographic reconstruction was carried out as described previously (Spilman et al., 2009). Tilt series were acquired semi-automatically using the FEI Xplore3D software at a magnification of $38,000 \times$ and nominal defocus of $6 \mu \mathrm{m}$ and collected over an angular range of up to $138^{\circ}$ in $2^{\circ}$ steps. The total electron dose for the entire tilt series was 80 to $120 \mathrm{e}^{-} / \AA^{2}$, or about 1 to $2 \mathrm{e}^{-} /$ $\AA^{2}$ for each image (McEwen et al., 1995). Images were recorded on a Gatan Ultrascan $4 \mathrm{k} \times 4 \mathrm{k}$ charge-coupled device camera operated in binned mode, resulting in a pixel size of $7.9 \AA$ on the sample. Tomographic data processing was done with the IMOD software package (IMOD 4.1.8, Boulder, CO; Kremer et al., 1996). No phase correction was used because all data used were within the first zero of the contrast transfer function (CTF), but a $22-\AA$ low-pass filter was applied during reconstruction. The casein micelle reconstructions were visualized using the University of California San Francisco Chimera visualization and analysis software package (Huang et al., 1996; Goddard et al., 2007). The 3-dimensional reconstruction was visualized at 2 density cutoff levels to differentiate between the protein and the colloidal nanoclusters. At 1.76 SD above the
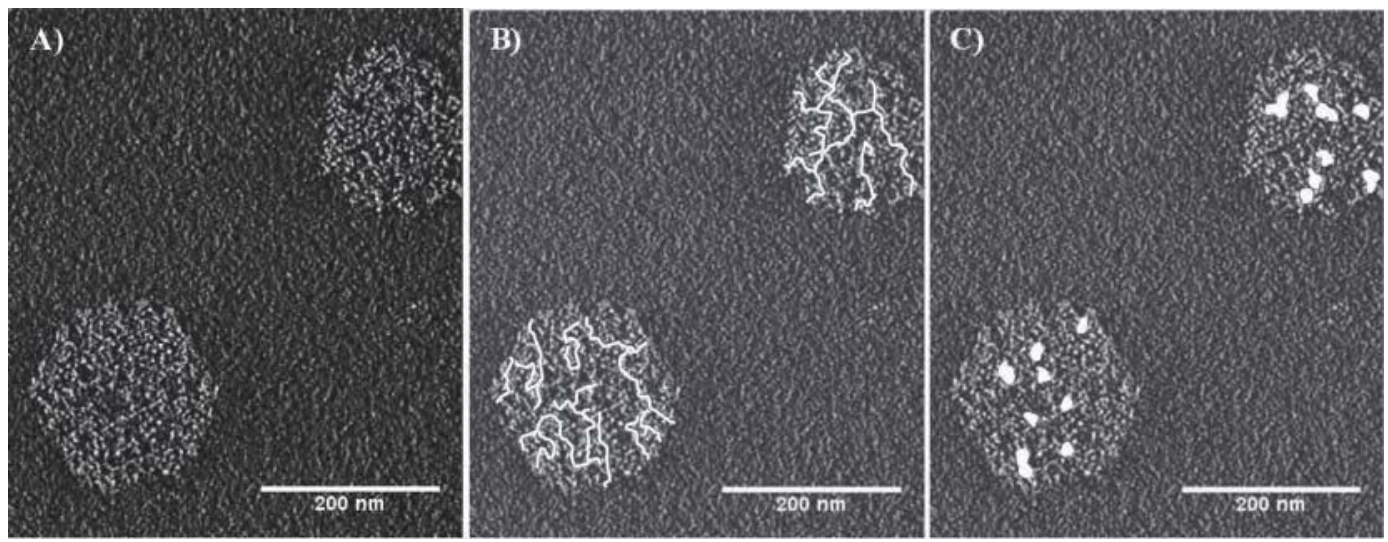

Figure 3. A) Slabs (4.74 nm) through the centers of 2 micelles from the cryo-transmission electron tomograms; B) highlighting of the internal channels in the native casein micelles in the same image; C) highlighting of the internal cavities in the native casein micelles in the same image. Scale bar $=200 \mathrm{~nm}$. 


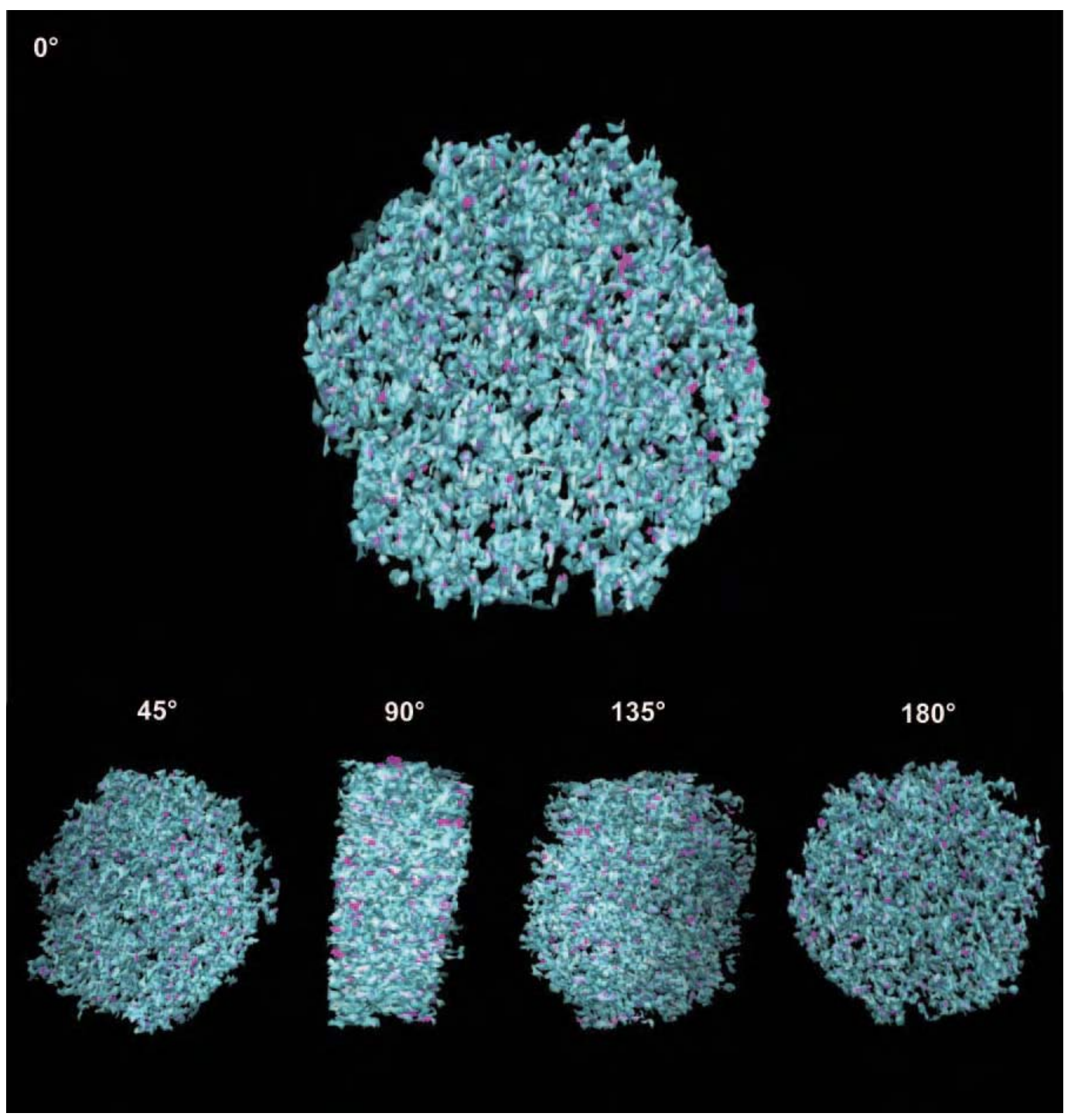

Figure 4. Isosurface representation of the tomogram of the native casein micelle, shown at 5 angles. The blue surface is shown at a cut-off level of $0.61 \mathrm{SD}$ above the mean, whereas the pink surface is at 1.76 SD above the mean, highlighting the positions of the higher density colloidal calcium phosphate. The transparency of the blue surfaces has been decreased to 0.45 to facilitate the viewing of the colloidal calcium phosphate (video file available at http://web.utk.edu/ fede/casein-micelle.html).

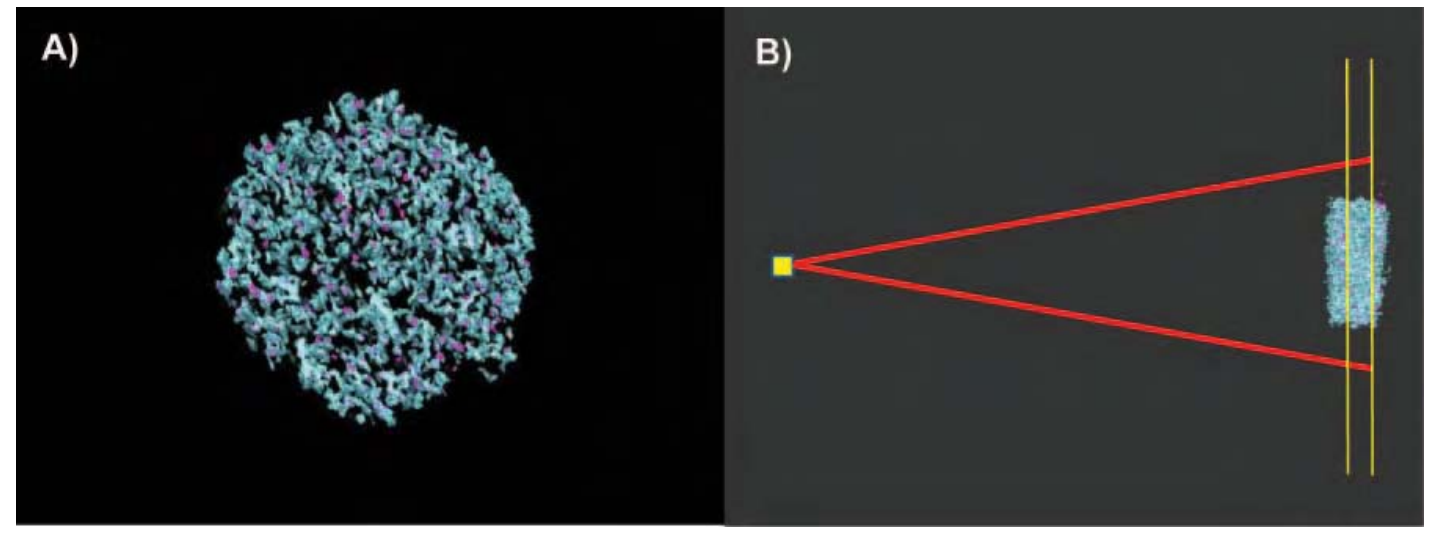

Figure 5. A) Isosurface representation of a 35-nm slab though the native casein micelle tomogram, showing the channels and cavities (black areas) found within the micelle; B) markers indicating the location of the slab within the interior of the casein micelle. 
mean, only the high-density colloidal nanoclusters were apparent. A Gaussian smoothing filter was applied and the map was visualized at $0.61 \mathrm{SD}$ above the mean to show the protein nanoclusters. The size of the calcium nanoclusters and the diameter of the internal structures of the casein micelle were measured using the Image $\mathrm{J}$ image analysis software package (ImageJ, National Institutes of Health, Bethesda, MD; http://imagej.nih. gov/ij/). The size and number of calcium nanoclusters was an average obtained from examining several casein micelles. The average diameter of the internal structures was calculated in the same manner. Video files showing unaligned and aligned micrographs, tomogram of casein micelles and casein micelle reconstruction are available at http://web.utk.edu/ fede/casein-micelle. html.

\section{RESULTS AND DISCUSSION}

A typical cryo-TEM image of the native casein micelles at $0^{\circ}$ tilt is shown in Figure 1. The 3 -dimensional tomogram was assembled from a series of such images at tilt angles from $-64^{\circ}$ to $+64^{\circ}$. A central section through one of the tomograms is shown in Figure 2. The cryo-TEM tomogram of the casein micelle shows that it is highly porous throughout its structure, as previously reported (McMahon and Oommen, 2008). The average thickness of the casein micelles in the tomograms was $100 \mathrm{~nm}$. The internal structures within the casein micelle exhibited a random arrangement, resulting in the presence of irregular water-filled channels larger than approximately $5 \mathrm{~nm}$ (Figure 3A and 3B) and inner cavities of 20 to $30 \mathrm{~nm}$ in diameter (Figure $3 \mathrm{~A}$ and $3 \mathrm{C}$ ). The existence of these structures has been previously suggested by Dalgleish (2011), who postulated that the hydrophobic interactions of the proteins would lead to an uneven distribution of water within the interior of the micelle and the formation of water channels. The existence of cavities within the micelle was also proposed by McMahon and Oommen (2008) as part of the interlocking lattice model of the casein micelle structure.

Isosurface representations of the 3-dimensional reconstruction are shown in Figure 4. The cylindrical appearance of the micelle is due to the deformation caused by the surface pressure of the 50- to 200-nmthick embedding film of vitreous ice, resulting in the spherical micelles being compressed into a more cylindrical shape. Nano-rheological studies of casein micelles using vibrating atomic force microscopy yielded Young's modulus of 0.06 to $0.08 \mathrm{MPa}$, typical of easily deformable polymeric soft foams (Helstad et al., 2005). This compressive deformation occurred parallel to the direction of observation and has been reported in previ- ous cryo-TEM studies of casein micelles (Marchin et al., 2007).

Colloidal calcium phosphate clusters (represented as pink particles in Figure 4) were found evenly distributed throughout the micelle with numbers ranging from 700 to 800 , as suggested by De Kruif and Holt (2003). The diameter of the CCP nanoclusters ranged from approximately 6 to $12 \mathrm{~nm}$, larger than the diameter $(4.8 \mathrm{~nm})$ reported by McMahon and Oommen (2008) but similar to that of the calcium phosphate aggregates observed by Hojou et al. (1977). An isosurface representation of a 35-nm-thick slab through the casein micelle reconstruction (Figure 5) shows the extensive number of water-filled channels and cavities within the inner structure of the native casein micelle.

\section{CONCLUSIONS}

In this study, cryo-TEM tomographic reconstruction allowed us to examine the interior of the native casein micelle. The tomograms demonstrated the presence of water-filled cavities and channels within the interior of the native micelle structure and the absence of any spherical protein substructures. These observations further question the existence of submicelles within the casein micelle, and confirm the existence of channels and inner cavities. These inner cavities, which are interconnected by wide channels, may explain the dynamic nature of the native casein micelle and its ability to release and retain $\beta$-casein and other macromolecules (Sahu et al., 2008).

\section{ACKNOWLEDGMENTS}

The 3-dimensional casein micelle images were produced using the UCSF Chimera package from the Resource for Biocomputing, Visualization, and Informatics at the University of California, San Francisco (supported by NIH P41 RR001081). This research was partially supported by the University of Tennessee Institute of Agriculture, Dairy Research Institute (Rosemont, IL), and the National Institutes of Health (project 1R21HD065170-01A1).

\section{REFERENCES}

Dalgleish, D. G. 2011. On the structural models of bovine casein micelles-Review and possible improvements. Soft Matter 7:22652272 .

De Kruif, C. G, and C. Holt. 2003. Casein micelle structure, functions and interactions. Pages 233-276 in Advanced Dairy Chemistry. Vol. 1. 3rd ed. P. F. Fox and P. L. H. McSweeney, ed. Kluwer Academic, New York, NY.

Dokland, T., and M. L. Ng. 2006. Transmission electron microscopy of biological specimens. Pages 153-211 in Techniques in Microscopy 
for Biomedical Applications. T. Dokland, D. W. Hutmacher, M. L. Ng, and J. T. Schantz, ed. World Scientific Press, Singapore.

Farrell, H. M., E. L. Malin, E. M. Brown, and P. X. Qi. 2006. Casein micelle structure: What can be learned from milk synthesis and structural biology? Curr. Opin. Colloid Interface Sci. 11:135-147.

Fox, P. F., and A. Brodkorb. 2008. The casein micelle: Historical aspects, current concepts and significance. Int. Dairy J. 18:677-684.

Goddard, T. D., C. C. Huang, and T. E. Ferrin. 2007. Visualizing density maps with UCSF Chimera. J. Struct. Biol. 157:281-287.

Hansen, S., R. Bauer, S. B. Lomholt, K. B. Quist, J. S. Pedersen, and K. Mortensen. 1996. Structure of casein micelles studied by smallangle neutron scattering. Eur. Biophys. J. 24:143-147.

Helstad, K. M., A. D. Bream, J. Trckova, M. Paulsson, and P. Dejmek. 2005. Nanorheological properties of casein. Spec. Publ. R. Soc. Chem. 298:218-229.

Hojou, K., T. Oikawa, K. Kanaya, T. Kimura, and K. Adachi. 1977. Some applications of ion-beam sputtering to high-resolution electron-microscopy. Micron 8:151-170.

Holt, C., C. G. De Kruif, R. Tuinier, and P. A. Timmins. 2003. Substructure of bovine casein micelles by small-angle x-ray and neutron scattering. Colloids Surf. A Physicochem. Eng. Asp. 213:275-284.

Horne, D. S. 1998. Casein interactions: Casting light on the black boxes, the structure in dairy products. Int. Dairy J. 8:171-177.

Horne, D. S. 2009. Casein micelle structure and stability. Pages 133162 in Milk Proteins: From Expression to Food. A. Thompson, M. Boland, and H. Singh, ed. Academic Press, New York. NY. Elsevier Inc.

Huang, C. C., G. S. Couch, E. F. Pettersen, and T. E. Ferrin. 1996. Chimera: An extensible molecular modeling application constructed using standard components. In Biocomputing: Proc. $1996 \mathrm{~Pa}-$ cific Symp. L. Hunter and T. Klein, ed. World Scientific Publishing Co., Singapore.

Kremer, J. R., D. N. Mastronarde, and J. R. McIntosh. 1996. Computer visualization of three-dimensional image data using IMOD. J. Struct. Biol. 116:71-76.
Marchin, S., J.-L. Putaux, F. Pignon, and J. Leonil. 2007. Effects of the environmental factors on the casein micelle structure studied by cryo transmission electron microscopy and small-angle $\mathrm{x}$ ray scattering/ultrasmall-angle x-ray scattering. J. Chem. Phys. 126:045101-045110.

McEwen, B. F., K. H. Downing, and R. M. Glaeser. 1995. The relevance of dose-fractionation in tomography of radiation-sensitive specimens. Ultramicroscopy 60:357-373.

McMahon, D. J., and B. S. Oommen. 2008. Supramolecular structure of the casein micelle. J. Dairy Sci. 91:1709-1721.

Nudelman, F., G. de With, and N. Sommerdijk. 2011. Cryo-electron tomography: 3-Dimensional imaging of soft matter. Soft Matter $7: 17-24$.

Qi, P. X. 2007. Studies of casein micelle structure: The past and the present. Lait 87:363-383.

Qi, P. X. 2009. Studies of the biological function and structure of casein micelles, and future implications. Woodhead Publ. Ser. Food Sci. Technol. Nutr. 182:147-169.

Sahu, A., N. Kasoju, and U. Bora. 2008. Fluorescence study of the curcumin-Casein micelle complexation and its application as a drug nanocarrier to cancer cells. Biomacromolecules 9:2905-2912.

Spilman, M. S., C. Welbon, E. Nelson, and T. Dokland. 2009. Cryoelectron tomography of porcine reproductive and respiratory syndrome virus: Organization of the nucleocapsid. J. Gen. Virol. 90:527-535.

Walstra, P. 1999. Casein sub-micelles: Do they exist? Int. Dairy J. 9:189-192.

Wong, D. W. S., W. M. Camirand, and A. E. Pavlath. 1996. Structures and functionalities of milk proteins. Crit. Rev. Food Sci. Nutr. $36: 807-844$. 